\title{
Modified culture system and combination of FICTION could increase the detection rate of abnormalities in multiple myeloma
}

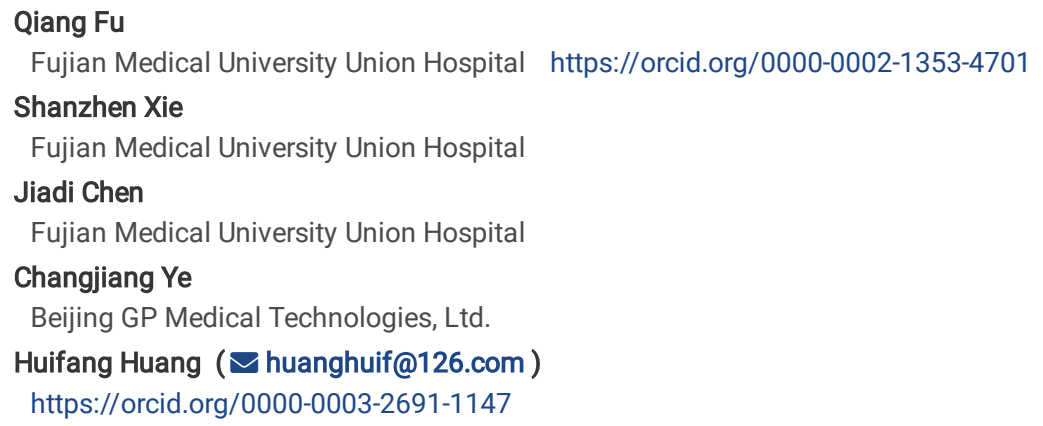

Research

Keywords: multiple myeloma, cytogenetic analysis, in situ hybridisation, fluorescence, immunophenotyping

Posted Date: June 23rd, 2020

DOI: https://doi.org/10.21203/rs.3.rs-34934/v1

License: (c) (7) This work is licensed under a Creative Commons Attribution 4.0 International License. Read Full License 


\section{Abstract \\ Background}

Conventional karyotyping of multiple myeloma (MM) is hampered by the low mitotic index of plasma cells (PCs). Furthermore, low proportions of PCs in some specimens may lead to false negative results in fluorescence in situ hybridisation (FISH) detection. The detection rate of cytogenetic abnormalities in MM must therefore be improved.

\section{Methods}

Bone marrow cells were cultured in an ordinary medium for $24 \mathrm{~h}$ or in a medium containing $10 \mathrm{ng} / \mathrm{mL}$ IL- 6 and $40 \mathrm{ng} / \mathrm{mL}$ GM-CSF for $6 \mathrm{~d}$. Fluorescence immunophenotyping and interphase cytogenetics as a tool for the investigation of neoplasms (FICTION) was also conducted, which combines fluorescence immunophenotyping (using CD138) and FISH.

\section{Results}

Under modified culture conditions, the successful rate of culture and abnormality rate during karyotype analysis increased to $86.4 \%$ and $40.9 \%$, respectively. The abnormality rate of FICTION (89.5\%) was significantly higher than that of FISH (60.0\%). The genetic abnormality rate increased to $92.3 \%$ when FICTION and karyotyping were conducted under modified culture conditions.

\section{Conclusion}

The established modified culture system could improve karyotyping quality in MM. Owing to its obvious advantages compared with FISH, FICTION is recommended for detecting genetic abnormalities in MM. Combining modified culture system and FICTION could improve the sensitivity of cytogenetic risk stratification in MM patients.

\section{Background}

Multiple myeloma (MM) is a heterogeneous disease associated with underlying chromosomal abnormalities and has greatly variable prognosis. Recent studies have shown that cytogenetic abnormalities are important factors affecting MM prognosis. However, MM karyotyping has been hampered not only by a low level of bone marrow (BM) infiltration and the low mitotic index of plasma cells (PCs) in vitro, but also the high viscosity of bone marrow fluid in MM patients, which often leads to specimen coagulation. In addition, in many MM cases, poor metaphase quality has prevented the identification of recurrent aberrations, such as translocations by chromosome banding. As a result, cytogenetic analysis of MM patients often fails, and the detection rate of abnormal karyotypes is low.

Extended culture time and cytokines such as granulocyte-macrophage colony- stimulating factor (GM-CSF), interleukin (IL)-3, IL-4, and IL-6 are generally applied to PC cultures to improve metaphase yield and increase the detection rate of cytogenetic abnormalities in MM [1-4]

Fluorescence in situ hybridisation (FISH) is the method of choice for fast and reliable identification of chromosomal alterations, including copy number abnormalities, genomic break points, and translocations or fusion genes. This method can also improve the detection rate of genetic abnormalities in MM. However, ordinary FISH may be unable to distinguish PCs from other nucleated cells when the proportion of PCs is low. As a result, the proportion of abnormal signals may be reduced, potentially resulting in false negative results. Fluorescence immunophenotyping and interphase hybridisation as a tool for the investigation of neoplasms (FICTION) is a technique that combines ordinary FISH and fluorescence immunophenotyping. The probe signals of the target cells can therefore be selectively analysed under a microscope. CD138 is usually employed to mark PCs, for it is almost $100 \%$ expressed in PCs, but not in BM hematopoietic progenitors and lymphocytes. Owing to its capacity to improve the efficacy of results interpretation, FICTION may be extremely useful in tumour diagnosis, particularly, for the detection of low tumour load, minimal residual disease (MRD), or composite tumour diagnosis [5].

The purpose of the current study was to assess the effect of cytokine stimulation and culture-time extension on the success and detection rates of genetic abnormalities in MM. In addition, the ability of FICTION to increase the detection rate of genetic abnormalities was investigated in this study.

\section{Results}

\section{Conventional cytogenetic analysis (CCA) of PCs cultured in improved conditions}

The analysis was double-blind. Ordinary culture served as a control. Both the number and quality of karyotypes available for analysis were significantly increased when BM cells were cultured in improved conditions by stimulating by cytokines and prolonging culture time (Fig. 1).

In the control group, chromosome analysis achieved a success rate of $77.3 \%$ (17/22), and 9.1\% (2/22) of abnormal karyotypes were detected. Both of the two abnormal karyotypes were complex (three or more abnormalities present). In the improved group, chromosome analysis achieved an $86.4 \%$ (19/22) success rate, and $40.9 \%$ (9/22) of abnormal karyotypes were detected. Of these abnormal karyotypes, $77.8 \%(7 / 9)$ were complex karyotypes. Abnormalities involved all chromosomes except chromosomes 16 and 22 (Table 1). All abnormalities detected in the control group were also found in the improved group. The detection rate of abnormal karyotypes in the improved group was significantly higher than that in the control group $(P=0.015)$.

Page 2/11 


\section{Table 1 CCA results in $22 \mathrm{MM}$ patients with and without improved culture conditions}

\begin{tabular}{|c|c|c|c|c|c|c|}
\hline No. & Gender & Age & $\begin{array}{l}\text { BM } \\
\text { nucleated } \\
\text { cell } \\
\text { proliferation }\end{array}$ & $\begin{array}{l}\text { PCs } \\
(\%)\end{array}$ & Control group & Experimental group \\
\hline 1 & Female & 35 & Active & 1.0 & $46, X X[7]$ & $46, X X[20]$ \\
\hline 2 & Male & 49 & Active & 74.5 & $46, X Y[2]$ & $46, \mathrm{XY},+3, \operatorname{del}(3)(\mathrm{p} 15 \mathrm{p} 23)[\mathrm{CP} 2] / 46, \mathrm{XY}[9]$ \\
\hline 3 & Female & 54 & Active & 75.0 & Failure & $\begin{array}{l}\text { 44-46,XX, del(1)(p11p22),ins(6;?)(p11;?),?inv(7) } \\
(\mathrm{q} 22 \mathrm{q} 36), \operatorname{add}(8)(\mathrm{p} 23), \operatorname{add}(9)(\mathrm{p} 13), \operatorname{add}(11)(\mathrm{p} 15), \operatorname{add}(14) \\
(\mathrm{q} 32),+\operatorname{mar}_{1},+\operatorname{mar}_{2}[\mathrm{CP} 15] / 80-88, \text { idem }^{*} 2[\mathrm{CP} 5]\end{array}$ \\
\hline 4 & Male & 69 & Active & 29.0 & $46, X Y[15]$ & $46, X Y[15]$ \\
\hline 5 & Male & 72 & Reduced & 52.5 & Failure & Failure \\
\hline 6 & Male & 63 & Active & 74.5 & $\begin{array}{l}52-61, \mathrm{XY},+3,+4,+5, \mathrm{i}(8)(\mathrm{q} 10),+9 \\
+9,+11,+15,+17,+21,+21,+\mathrm{mar}_{1} \\
+\operatorname{mar}_{2}[\mathrm{CP} 3] / 46, \mathrm{XY}[2]\end{array}$ & $\begin{array}{l}44-63, X Y,+2,+4,+5,+5,+7, \text { add }(7) \\
(p 22),+9,+9,+11,+11,+17,+19,+21,+21 \\
{[C P 9] / 46, X Y[11]}\end{array}$ \\
\hline 8 & Male & 41 & Active & 26.5 & $46, \mathrm{XY}[7]$ & $\begin{array}{l}\text { 46-48,XY,add(2)(p36),-4,add(6)(q21), add(6) } \\
(\mathrm{q} 23),-12, \operatorname{add}(14)(\mathrm{q} 32), \operatorname{add}(17)(\mathrm{p} 11), \mathrm{del}(18) \\
(\mathrm{q} 21),+\operatorname{mar} 1 * 2,+\operatorname{mar} 2,+\operatorname{mar} 3,+\operatorname{mar} 4[\mathrm{CP} 11] / 46, \mathrm{XY}[5]\end{array}$ \\
\hline 9 & Male & 74 & Active & 26.5 & $46, X Y[20]$ & $\begin{array}{l}\text { 50-53,XY,del(1)(q12),+4,+5, del(8)(p21),+10,del(10) } \\
\text { (p12),+11,+11,del(11)(q23), +12,del(12)(p12),+13,add(21) } \\
\text { (q22),+mar[CP5]/46,XY[15] }\end{array}$ \\
\hline 10 & Male & 53 & Active & 78.0 & $46, \mathrm{XY}[5]$ & Failure \\
\hline 11 & Female & 53 & Active & 53.5 & $46, X X[20]$ & $46, X X,+$ mar,inc $[C P 3] / 46, X X[4]$ \\
\hline 12 & Male & 57 & Active & 1.0 & Failure & $46, \mathrm{XY}[2]$ \\
\hline 13 & Male & 57 & Active & 1.0 & $46, X Y[20]$ & $46, X Y[20]$ \\
\hline 14 & Male & 68 & $\begin{array}{l}\text { Extremely } \\
\text { active }\end{array}$ & 55.5 & $46, X Y[20]$ & $46, X Y[20]$ \\
\hline 15 & Male & 88 & Active & 40.0 & Failure & Failure \\
\hline 16 & Male & 59 & Reduced & 7.0 & $46, X Y[5]$ & $46, X Y[15]$ \\
\hline 17 & Female & 50 & Active & 41.5 & $46, \mathrm{XY}[12]$ & $46, X Y[14]$ \\
\hline 18 & Male & 57 & Active & 65.0 & $46, \mathrm{XY}[9]$ & $42,+$ mar,inc[CP2]/46,XY[2] \\
\hline 19 & Male & 62 & Reduced & 10.0 & Failure & $\begin{array}{l}\text { 36-39,?dic(1;11)(q10;q13), add(6)(q11),?del(15) } \\
\text { (q14q23), add(19)(p13), +mar } 1,+ \text { mar }_{2},+ \text { mar }_{3}, \text { inc[CP2] }\end{array}$ \\
\hline 20 & Female & 68 & Active & 12.0 & $\begin{array}{l}\text { 41-43,t(13;22) } \\
\text { (q32; } 11), \operatorname{add}(14) \\
\text { (q32),add(20) } \\
\text { (p13),+mar,inc[CP2]/46,XX[18] }\end{array}$ & $\begin{array}{l}\text { 44-45,X,-X,+9,t(13;22)(q32;q11), add(14)(q32), add(19)(p13), } \\
\operatorname{der}(20) t(1 ; 20)(q 11 ; p 13),+\operatorname{mar}[C P 4] / 46, X X[16]\end{array}$ \\
\hline 21 & Male & 61 & Active & 0 & $46, \mathrm{XY}[20]$ & $46, \mathrm{XY}[20]$ \\
\hline 22 & Female & 62 & Active & 10.0 & $46, X X[20]$ & $46, X X[20]$ \\
\hline 23 & Male & 58 & Active & 2.0 & $46, X Y[2]$ & $46, X Y[10]$ \\
\hline
\end{tabular}

CCA, conventional cytogenetic analysis; MM, multiple myeloma; BM, bone marrow; PCs, plasma cells.

\section{Panel FISH and FICTION}

Chromosomal abnormalities were detected by FISH in 15 (60.0\%) of 25 patients. The highest detection rate was observed for $/ g H$ break-apart (signal patterns including 101R1G, 101R, 101R2G, and 1R), with a positive rate of 40.0\%. The detection rates of 1q21 amplification, RB1 deletion, D13S319 deletion, and P53 deletion were $32.0 \%, 28.0 \%, 20.0 \%$, and $4.0 \%$, respectively. Some cells manifested $I g H$ deletion (10) in one specimen, and in another, some cells exhibited $I g H$ amplification (30). 
Chromosomal abnormalities were detected in 17 (89.5\%) of 19 patients by FICTION, and all abnormalities detected by FISH were also found by FICTION (Fig. 2). The highest positive rate was observed for detecting $R B 1$ deletion, with a positive rate of $73.7 \%$. The positive rates of $I g H$ break-apart (signal patterns including 101R1G, 101R, 201R, 101R2G, and 1R), 1q21 amplification, D13S319 deletion, and P53 deletion were 68.4\%, 57.9\%, 57.9\%, and 42.1\%, respectively. Two samples manifested 1q21 deletion (1R) and IgH deletion (10), and one sample manifested IgH amplification (30).

The abnormal detection rates of each probe are shown in Fig. 3. Significant differences in the abnormal rate were found between FISH and FICTION when detecting $R B 1$, D13S319, and $P 53$, with $P$ values of $0.003,0.013$, and 0.006 , respectively.

Of the seventeen abnormal specimens detected by FICTION, only one patient (patient No. 8) yielded results consistent with those of FISH. However, the proportion of positive cells was higher than in FISH. 1 q21 amplification yielded $60.0 \%$ and $18.0 \%$ positive cells in FICTION and FISH, respectively, and IgH break-apart yielded $38.0 \%$ and $15.0 \%$. The other 16 cases with inconsistent results are shown in Table 2. The abnormal detection rate of FICTION (89.5\%) was significantly higher than that of $\mathrm{FISH}(60.0 \%)(P=0.030)$.

Table 2

16 cases with inconsistent results between FISH and FICTION

\begin{tabular}{|c|c|c|c|c|c|c|c|c|c|c|c|}
\hline \multirow[t]{2}{*}{ No. } & \multirow[t]{2}{*}{$\mathrm{PCs}(\%)$} & \multicolumn{5}{|c|}{ Abnormality detection rates of FISH(\%) } & \multicolumn{5}{|c|}{ Abnormality detection rates of FICTION(\%) } \\
\hline & & 1q21(R) & RB1(G) & D13S319(R) & P53(G) & $\lg \mathrm{H}$ & 1q21(R) & RB1(G) & D13S319(R) & P53(G) & $\mathrm{IgH}$ \\
\hline 3 & 75.0 & $\mathrm{~N}$ & 36 & 22.5 & $\mathrm{~N}$ & $12(1 \mathrm{R})$ & 14 & 52 & 36.5 & $\mathrm{~N}$ & $52(1 \mathrm{R})$ \\
\hline 4 & 29.0 & $\mathrm{~N}$ & & & & & 30 & 48.5 & 28 & 28 & 30.5 \\
\hline 5 & 52.5 & $\mathrm{~N}$ & & & & & $\mathrm{~N}$ & 52 & $\mathrm{~N}$ & $\mathrm{~N}$ & $\mathrm{~N}$ \\
\hline 7 & 27.0 & $\mathrm{~N}$ & $\mathrm{~N}$ & $\mathrm{~N}$ & $\mathrm{~N}$ & 11(101R1G) & 46.5 & 28.5 & $\mathrm{~N}$ & $\mathrm{~N}$ & 50 \\
\hline 9 & 26.5 & $\mathrm{~N}$ & $\mathrm{~N}$ & $\mathrm{~N}$ & $\mathrm{~N}$ & 21.5(101R2G) & $22.5(1 \mathrm{R})$ & 42.5 & 30 & $\mathrm{~N}$ & $55.5\left(1 \mathrm{R}^{-}\right.$ \\
\hline 10 & 78.0 & $\mathrm{~N}$ & 73.5 & 79 & 76.5 & 44 & 33 & 78 & 82 & 70.5 & 95 \\
\hline 14 & 55.5 & $\mathrm{~N}$ & 48 & 51.5 & $\mathrm{~N}$ & 45.5 & $36.5(1 R)$ & 40.5 & 70.5 & $\mathrm{~N}$ & 47 \\
\hline 15 & 40.0 & $\mathrm{~N}$ & & & & & $\mathrm{~N}$ & 36 & 34.5 & 28 & $34.5\left(1 \mathrm{R}^{-}\right.$ \\
\hline 19 & 10.0 & $\mathrm{~N}$ & & & & & 37 & 34.5 & 20 & $\mathrm{~N}$ & 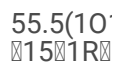 \\
\hline 20 & 12.0 & $14(3 R)$ & 14.5 & $\mathrm{~N}$ & $\mathrm{~N}$ & $13(10)$ & 42.5 & 74.5 & 17 & $\mathrm{~N}$ & 42.5(10) \\
\hline 21 & 0 & $\mathrm{~N}$ & $\mathrm{~N}$ & $\mathrm{~N}$ & $\mathrm{~N}$ & $12(30)$ & 12.5 & 12.5 & $\mathrm{~N}$ & $\mathrm{~N}$ & 18(30) \\
\hline 23 & 2.0 & $\mathrm{~N}$ & & & & & $\mathrm{~N}$ & $\mathrm{~N}$ & $\mathrm{~N}$ & 18 & 26 \\
\hline 25 & 6.5 & 8.5 & $\mathrm{~N}$ & 13.5 & $\mathrm{~N}$ & $\mathrm{~N}$ & 28 & 24 & 30.5 & 32.5 & 23.5 \\
\hline 26 & 24.5 & $\mathrm{~N}$ & & & & & $\mathrm{~N}$ & $\mathrm{~N}$ & 10 & 14 & $\mathrm{~N}$ \\
\hline 27 & 27.0 & 8 & $\mathrm{~N}$ & 19.5 & $\mathrm{~N}$ & $\mathrm{~N}$ & $23.5(3 \mathrm{R})$ & 36 & 32 & 23.5 & $\mathrm{~N}$ \\
\hline 28 & 57.0 & $\mathrm{~N}$ & $\mathrm{~N}$ & $\mathrm{~N}$ & $\mathrm{~N}$ & 24.5 & 29.5 & 46 & $\mathrm{~N}$ & 36.5 & 55.5 \\
\hline
\end{tabular}

FISH, fluorescence in situ hybridisation; FICTION, fluorescence immunophenotyping and interphase cytogenetics as a tool for the investigation of neoplasms; cells; N, normal; R, red; G, green; O, orange.

Four of the 15 (26.7\%) chromosomal abnormalities detected by FISH had only one chromosomal abnormality; 11 of 15 (73.3\%) had more than two chromosomal abnormalities. Of the 17 samples with chromosomal abnormalities detected by FICTION, only one case (5.9\%) had one chromosomal abnormality. The other 16 cases $(94.1 \%)$ had more than two chromosomal abnormalities. The rate of complex karyotype detection therefore increased significantly.

As shown in Table 3, the median proportion of abnormal positive cells detected by FICTION was higher compared to that by FISH, and the median proportion of PCs was lower in patients with chromosomal abnormalities. In 11 cases, abnormal clones were detected by both FISH and FICTION. Four other cases were determined to be abnormal via FICTION, but the proportion of abnormal clone-positive cells detected by FISH was a critical value. In these 15 samples, a high proportion of abnormal cells were detected by FICTION (Fig. 4). The differences between the positive rates of 1q21, RB1, D13s319, and IgH detection were statistically significant, with $P$ values of $0,0.038,0.004$, and 0.001 , respectively. 
Table 3

FISH and FICTION detection of common chromosome abnormalities and positive cell ratio

\begin{tabular}{|c|c|c|c|c|c|}
\hline \multirow[t]{2}{*}{ Probe } & \multirow{2}{*}{$\begin{array}{l}\text { Signal } \\
\text { pattern }\end{array}$} & \multicolumn{2}{|l|}{ FISH(\%) } & \multicolumn{2}{|l|}{ FICTION(\%) } \\
\hline & & $\begin{array}{l}\text { Positive cell proportion } \\
\text { range (Median) }\end{array}$ & $\begin{array}{l}\text { PC proportion range of positive } \\
\text { sample (Median) }\end{array}$ & $\begin{array}{l}\text { Positive cell proportion } \\
\text { range (Median) }\end{array}$ & $\begin{array}{l}\text { PC proportion range of positive } \\
\text { sample (Median) }\end{array}$ \\
\hline \multirow[t]{3}{*}{$1 q 21$} & $3 R$ & $8-14(8)$ & \multirow[t]{3}{*}{$1-65(27)$} & $12-46(29.5)$ & \multirow[t]{3}{*}{$0-78(27)$} \\
\hline & $4 \mathrm{R}$ & $18(/)$ & & $60(/)$ & \\
\hline & $1 \mathrm{R}$ & / & & $22-36(29)$ & \\
\hline$R B 1$ & $1 \mathrm{G}$ & $14-73(42)$ & $12-78(50.75)$ & $12-78(41)$ & $0-78(34.5)$ \\
\hline D13S319 & $1 \mathrm{R}$ & $13-79(22)$ & $6.5-78(55.5)$ & $10-82(30)$ & $6.5-78(27)$ \\
\hline P53 & $1 G$ & $76(/)$ & $78(/)$ & $14-70(28)$ & $2-78(33.5)$ \\
\hline \multirow[t]{7}{*}{$\operatorname{lgH}$} & 101R1G & $11-45(34)$ & \multirow[t]{7}{*}{$12-78(55.5)$} & $18-95(40.5)$ & \multirow[t]{7}{*}{$0-78(28)$} \\
\hline & 101R2G & $21(/)$ & & $55(/)$ & \\
\hline & 101R & $15(/)$ & & $11-38(24.5)$ & \\
\hline & 201R & / & & $15.5(/)$ & \\
\hline & $1 \mathrm{R}$ & $12(/)$ & & $15-52(33.5)$ & \\
\hline & 30 & $12(/)$ & & $18(/)$ & \\
\hline & 10 & $13(/)$ & & $14-42(28)$ & \\
\hline
\end{tabular}

\section{Genetic abnormality detection rate of FISH with traditional CCA compared with FICTION with improved CCA}

Sixteen of 25 (64.0\%) genetic abnormalities were detected by FISH combined with traditional CCA, while 12 of 13 (92.3\%) were detected by FICTION combined with improved CCA. The latter had a significantly increased detection rate for genetic abnormalities. Further, the difference between the two groups was significant $(P<0.05)$.

\section{Discussion}

Genetic abnormality is an important factor for risk stratification in MM [6], as well as for predicting survival and defining a treatment strategy. It has been reported that almost all MM patients possess cytogenetic abnormalities, sometimes during the course of the disease [7]. However, PCs occur at the final stage of differentiation, which have a low proliferation rate and long cell cycle. The viscosity of bone marrow fluid in MM patients is high, which often causes specimens to coagulate during extraction. As a result, cytogenetic studies of $\mathrm{MM}$ after conventional short-term culture methods are hampered by difficulty in or even failure to obtain good metaphases for analysis. Furthermore, the abnormal detection rate of karyotypes is also low. In previously reported studies [8$10]$, only $20-40 \%$ of abnormal karyotypes could be detected by traditional metaphase cytogenetic analysis.

To improve the detection of chromosomal abnormalities in MM, the application of different cytokines was explored. IL-6 has been recognised as the major growth factor for PCs both in vitro and in vivo. It has been used in combination with haematopoietic factors such as GM-CSF and IL-3, as these growth factors increase the responsiveness of IL-6 for supporting PC growth. However, controversial results have been reported. Lai[4], Facon[2], and Cuneo [1] have suggested that adding different combinations of GM-CSF, IL-6, and IL-3 to the culture medium and prolonging culture time to $6 \mathrm{~d}$ offers an advantage over unstimulated overnight cultures. In contrast, Smadja [11] and Brigaudeau [12] found that compared to adding growth factors, the abnormal detection rate of a 3-day unstimulated culture was comparable or higher.

Based on previous studies, IL-6 $(10 \mathrm{ng} / \mathrm{mL})$ and GM-CSF $(40 \mathrm{ng} / \mathrm{mL})$ were added to the culture medium in this study and prolonged the culture time to $6 \mathrm{~d}$ to improve the abnormal detection rate of karyotypes in MM patients. As a result, the number of metaphases and the quality of chromosomes improved significantly in most samples compared to ordinary CCA. However, abnormal karyotypes were detected in samples 10 and 17 under improved culture conditions, while the conventional short-term culture method detected normal karyotypes. The number of karyotypes was lower after culture in improved conditions. This may be because the proliferation of non-PCs is faster during short-term culture, and PC proliferation inhibits that of non-PCs after the addition of IL-6 and GM-CSF.

Compared with conventional culture, culturing in improved conditions increased the karyotype and abnormal karyotype detection rates of MM patients. The abnormal detection rate reached $40.9 \%$, which was similar to that reported in the literature $[2,4,10]$.

It is now recognised that there are two broad genetic subtypes of $\mathrm{MM}$, as defined by chromosome number. Hyperdiploid myeloma (48-74 chromosomes) accounts for about $50 \%$ of cases and are mostly trisomies of chromosomes $3,5,7,9,11,15,19$, and 21 . This subtype rarely involves $/ g H$ translocations at the 
locus 14q32. Non-hyperdiploid myeloma (< 48 or $>74$ chromosomes) is associated with the presence of IgH translocations [13]. This study confirms and expands upon prior reports, identifying that 2 cases of hyperdiploidy and 7 cases of non-hyperdiploidy were detected in improved culture conditions. Further, $88.9 \%(8 / 9)$ had both numerical and structural abnormalities. This suggested that the chromosomal abnormalities in MM were often complex. The abnormality involves all chromosomes except chromosomes 16 and 22, as abnormal hyperdiploid MM typically involves chromosomes 2, 4, 5, 7, 9, 10, 11, 12, $13,17,19$, and 21 . Three cases of non-hyperdiploid MM were associated with $/ g H$ translocation and 2 cases with structural abnormalities involving $19 p 13$.

A considerable proportion of MM patients have only a small proportion and uneven distribution of PCs in the BM. Therefore, determining a means by which to accurately identify PCs under a microscope without the interference of normal cells is the primary issue to be addressed. The International Myeloma Working Group and the European Myeloma Network recommend that FISH detection in MM should not be conducted without first concentrating the PCs or employing a means for identifying PCs, ensuring that only these cells are scored [14] To fulfil this recommendation, PCs need to be sorted by CD138 immunomagnetic bead-based sorting or by labelling the cytoplasmic immunoglobulin light chain (clg).

However, enriching PCs via CD138 immunomagnetic bead-based sorting requires at least $20 \mathrm{~mL}$ [15] to satisfy the detection requirements, specimen acquisition is difficult, and the sorting process is labour and time-intensive. Cytoplasmic light chain immunofluorescence combined with FISH (clg-FISH) requires identification of the light chain types of clonal PCs before detection, ensuring the correct light chain antibody is selected. This is not easily incorporated into the routine workflow of a cytogenetic laboratory. Another proposed technique is the use of an initial Giemsa stain with PC mapping by image analysis software, followed by subsequent FISH analysis of the previously mapped PCs [16]. However, this technique relies on expensive image analysis software, which inevitably limits its clinical application.

Unlike the techniques mentioned above, when used in combination with simultaneous immunofluorescence (IF) using CD138 and FISH, FICTION does not require a large number of PCs, nor prior knowledge of the restricted light chain involved. Further, specialised procedures such as cell sorting or image analysis are unnecessary for this method. This makes it possible to recognise PCs under a fluorescence microscope and interpret genetic changes in PCs with cytoplasmic staining. FICTION can be implemented in laboratories that routinely perform FISH analysis, which is suitable for both fresh BM specimens and archived BM smears [16].

In the present study, FICTION detected a higher percentage of abnormal cases than FISH. In one CR sample (No. 21), the short-term conventional culture method, CCA with the improved culture system, and FISH did not detect abnormalities, but FICTION detected a low proportion of positive signals. These results suggested that the patient may be in the early stages of relapse. Compared to conventional FISH analysis, FICTION is more sensitive and could detect abnormalities when the proportion of PCs was low. FICTION is also more specific, detecting a higher proportion of abnormal cells compared with FISH when the proportion of PCs was the same.

Hanamura [17] showed that the 1q21 amplification rate of newly diagnosed MM patients is less than $20 \%$, while the detection rate of relapsed patients can increase to $44 \%$. Patient prognosis is worse when increased amplification signals are detected. In the current study, $41 \mathrm{q} 21$ amplification signals were detected by FICTION in patient No. 854 months after diagnosis. FICTION detected $60 \%$ positive cells, while FISH detected only $18 \%$. Two months after the detection of 4 amplified signals in 1q21, the patient discontinued treatment due to septic shock, paroxysmal atrial fibrillation, cardiac insufficiency, decompensated respiratory alkalosis, coagulation abnormalities, and electrolyte disturbance.

A partial deletion of chromosome 13 is the first genetic abnormality found in MM and is related to drug resistance. In terms of prognosis, $13 / 13 q$ abnormalities are often attributed to a poor outcome, regardless of whether they receive conventional chemotherapy or autologous stem cell transplantation [18]. The positive rate of $R B 1$ and D13S319 detection by FISH was higher in this study than those reported in the literature [17]. This may be related to different methodologies and was also affected by the small sample size.

This study determined that combining CCA with improved culture conditions and FICTION could increase the abnormality detection rate to $92.3 \%$. Combining CCA with improved culture conditions and FICTION could improve the sensitivity of cytogenetic risk stratification in MM patients, allow clear interpretation of the results of numerical critical sample detection, and provide more accurate information for personalised medicine. In future, the number of samples will be expanded to confirm the difference in detection quality between the two methods.

\section{Conclusion:}

The established modified culture system could improve karyotyping quality in MM. Owing to its obvious advantages compared with FISH, FICTION is recommended for detecting genetic abnormalities in MM. Combining modified culture system and FICTION could improve the sensitivity of cytogenetic risk stratification in MM patients.

\section{Materials And Methods}

\section{Patient selection and basic characteristics}

BM specimens were extracted from 28 MM patients who visited Fujian Medical University Union Hospital from January through December 2018. The criteria of "Hematology Diagnosis and Efficacy Standards Third Edition" by Zhinan Zhang [19] were used to establish the diagnosis, and all cases fulfilled the criteria for MM. There were 21 males and 7 females, with a median age of 58 years (range, 35-88 years) and median BM PC percentage of $27 \%$ (range, $0-78 \%$ ). The monoclonal component was IgA in 7 patients, IgD in 2 patients, IgG in 15 patients, and Bence-Jones protein in 4 patients. The staging was done according to the international staging system (ISS). Five patients were in stage I, 2 were in stage II, 20 were in stage III, and 1 was in complete remission (CR). Nineteen patients were newly diagnosed, and nine were revisited. A bone aspirate smear of each patient was stained with Wright-Giemsa. At least 200 cells were

Page 6/11 
counted for cell classification, and the PC proportion was calculated. Due to the limited number of specimens in some patients, it was impossible to complete one of the cytogenetic analytic techniques, either FISH or FICTION. Therefore, the numbers of cases in each group were slightly different. The study was approved by the ethics committee of Fujian Medical University Union Hospital (Ethics protocol number: 2016KY050), and BM samples were collected after obtaining written informed consent from each patient.

\section{CCA}

CCA was performed on 24-h BM cultures [20]. At least 20 metaphases or all available metaphases were examined. The International System for Cytogenetic Nomenclature (2013) was used to describe abnormal clones. Each specimen was analysed by at least two experienced laboratories.

\section{Effect of stimulating PCs with cytokines and extending culture time on CCA}

Nucleated cells $\left(2 \times 10^{6} / \mathrm{mL}\right)$ from BM were seeded into $5 \mathrm{~mL}$ RPMI 1640 (Hyclone, USA) medium containing $10 \%$ foetal calf serum, followed by the addition of IL-6 (10 ng/mL, PeproTech, USA) and GM-CSF ( $40 \mathrm{ng} / \mathrm{mL}$, PeproTech). Cells were cultured at $37^{\circ} \mathrm{C}$ under $5 \% \mathrm{CO}_{2}$ conditions for $6 \mathrm{~d}$, followed by harvesting. Cytogenetic analyses were carried out as above.

\section{FISH analysis}

MM detection kit was provided by GP Medical Technologies (Beijing, China, http://www.gpmedical.com.cn/), with a probe panel consisting of 1q21 (red), RB1 (13q14, green), D13S319 (13q14.3, red), P53 (17p13.1, green), and IgH break-apart probe (14q32, green and red fusion/orange). Uncultured BM cell suspension was dropped onto glass slides, and the next steps were followed as per the manufacturer's instructions. BM specimens from normal donors served as controls. The percentage of fluorescence-positive cells $\geq$ the mean \pm 3 standard deviations for the controls was applied as a positive criterion. The threshold was $6.6 \%$ for $1 \mathrm{q} 21$ amplification, $6.45 \%$ for $R B 1$ deletion, $6.95 \%$ for D13S319 deletion, and $8.25 \%$ for $P 53$ deletion. A break was defined in the $I g H$ break-apart probe when a red/green or orange fusion signal $(0)$ was split into separate red and green signals. Only red and green signals, which were more than one signal diameter apart from each other, were counted as a break. The threshold for $1 g H$ break-apart was $6.85 \%$. Fluorescent hybridisation signals were counted in 200 cells underneath a fluorescence microscope (Olympus BX51, Tokyo, Japan) using an oil immersion objective and filter sets for DAPI, FITC, and RHOD. Raw fluorescence images were analysed using IMSTAR FISH 2.1 (GP Medical Technologies).

\section{FICTION analysis}

Red blood cells from uncultured BM were fully lysed with red blood cell lysis buffer (Genview, USA). Pre-fixed cell suspension was mixed with - $20{ }^{\circ} \mathrm{C}$ precooled methanol (1:1 cell suspension: methanol). BM cell suspension was dropped onto glass slides, and the slides were air-dried. The slides were then fixed with Triton 100-X/4\% PFA (1:1, 0.5\% Triton X-100: 4\% PFA) for 10 min. The following steps were the same as those carried out in ordinary FISH. The glass slides were washed and dehydrated in gradient ethanol solutions (70\%, 85\%, and 100\%) after hybridisation. They were then blocked with $2.0 \%$ BSA for 5 min, and mouse anti-human CD138 antibody (primary antibody) and AMCA-labelled goat anti-mouse lgH antibody (secondary antibody) (PeproTech) were added. The slides were incubated at room temperature and protected from light for $1 \mathrm{~h}$. The samples were air-dried in the dark, and 10 $\mu \mathrm{L} 4$ ',6-diamidino-2phenylindole (DAPI) counterstain was added to each. Slides were covered with a coverslip and stained for 5 min. Using an oil immersion objective and filter sets for DAPI, AQUA, FITC, and RHOD, FISH signals were analysed only in cells with blue cytoplasm. At least 200 cells were counted. When the percentage of PCs in BM was low, at least 50 cells were counted.

\section{Statistical analysis}

Fisher's test was used for comparing the detection rate of karyotypic abnormalities between the two groups. Chi-square test was applied for analysing the differences in the abnormal detection rate of each probe. The number of positive cells detected by FISH and FICTION were compared between groups with a paired t-test. All analyses were performed using SPSS 25.0 Statistics (SPSS Inc., Chicago, IL, USA).

\section{Abbreviations}

MM: multiple myeloma

BM: bone marrow

PC: plasma cell

GM-CSF: granulocyte-macrophage colony- stimulating factor

IL: interleukin

CCA: Conventional cytogenetic analysis

FISH: Fluorescence in situ hybridization

FICTION: Fluorescence immunophenotyping and interphase hybridisation as a tool for the investigation of neoplasms

MRD: minimal residual disease

ISS: international staging system

CR: complete remission 


\section{Declarations}

\section{Ethics approval and consent to participate}

The study was approved by the Ethics Committee of Fujian Medical University Union Hospital, and bone marrow samples were collected after obtaining written informed consent from each patient.

\section{Consent for publication}

All patients in this study provided their consent for publication.

\section{Availability of data and materials}

All data generated or analyzed during this study are included in this published article and its additional files.

\section{Competing interests}

The authors declare that they have no competing financial interests.

\section{Funding}

This study was sponsored by National and Fujian Provincial Key Clinical Specialty Discipline Construction Program, P. R.C, Construction project of Fujian medical center of hematology (Min201704), and Startup Fund for scientific research, Fujian Medical University (2016QH031 ).

\section{Authors' Contributions}

Qiang Fu and Huifang Huang designed the study. Clinical data collection and FICTION were performed by Qiang Fu and Shanzhen Xie. FISH and cell culture were performed by Jiadi Chen and Changjiang Ye. Karyotype analysis was performed by Qiang Fu and Huifang Huang. The first draft of the manuscript was written by Qiang Fu and Shanzhen Xie, and all authors commented on previous versions of the manuscript. All authors read and approved the final manuscript.

\section{Acknowledgements}

We would like to thank all the patients and family members participating in this work for their cooperation. This study was supported by Fujian Medical University Union Hospital. We appreciate the financial support of National and Fujian Provincial Key Clinical Specialty Discipline Construction Program, P. R.C, Construction project of Fujian medical center of hematology (Min201704), and Startup Fund for scientific research, Fujian Medical University ( Grant number: 2016QH031).

\section{References}

1. Cuneo A. Balsamo, et al. Interleukin-3 plus interleukin-6 may improve chromosomal analysis of multiple myeloma: Cytologic and cytogenetic evidence in thirty-four patients. Cancer Genet Cytogenet. 1996;90(2):171-5. https://doi.org/10.1016/s0165-4608(96)00129-x.

2. Facon T, Lai JL, Nataf E, et al. Improved cytogenetic analysis of bone marrow plasma cells after cytokine stimulation in multiple myeloma: a report on 46 patients. Br J Haematol. 1993;84:743-5. https://doi.org/ 10.1111/j.1365-2141.1993.tb03155.x.

3. HernÁndez G. Almeida, et al. IL-4 improves the detection of cytogenetic abnormalities in multiple myeloma and increases the proportion of clonally abnormal metaphases. Br J Haematol. 1998;103:163-7. https://doi.org/ 10.1046/j.1365-2141.1998.00951.x.

4. Lai JL, Zandecki M, Mary JY, et al. Improved cytogenetics in multiple myeloma: a study of 151 patients including 117 patients at diagnosis. Blood. 1995;85(9):2490-7.

5. Martínez-Ramírez A, Cigudosa JC, Maestre L, et al. Simultaneous detection of the immunophenotypic markers and genetic aberrations on routinely processed paraffin sections of lymphoma samples by means of the FICTION technique. Leukemia. 2004;18(2):348-53. https://doi.org/ $10.1038 /$ sj.leu.2403230

6. Stewart AK, Bergsagel PL, Greipp PR, et al. A practical guide to defining high-risk myeloma for clinical trials, patient counseling and choice of therapy. Leukemia. 2007;21(3):529-34. https://doi.org/ 10.1038/sj.leu.2404516.

7. Yuregir OO, Sahin Fl, Yilmaz Z, et al. Fluorescent in situ hybridization studies in multiple myeloma. Hematology. 2009;14(2):90-4. https://doi.org/ $10.1179 / 102453309 \times 385250$.

8. Dong H, Yang HS, Jagannath S, et al. Risk stratification of plasma cell neoplasm: insights from plasma cell-specific cytoplasmic immunoglobulin fluorescence in situ hybridization (clg-FISH) vs. conventional FISH. Clin Lymphoma Myeloma Leuk. 2012;12(5):366-74. https://doi.org/ 10.1016/j.clml.2012.05.003.

9. Lai YY, Huang XJ, Cai Z, et al. Prognostic power of abnormal cytogenetics for multiple myeloma: a multicenter study in China. Chin Med J (Engl). 2012;125(15):2663-70.

10. Cun-Bang W, Jing W, Ke Y, et al. Retrospective Analysis of Genetics Abnormalities in Patients with Multiple Myeloma. Zhongguo Shi Yan Xue Ye Xue ZA Zhi. 2018;26(6):1681-7. https://doi.org/ 10.7534/j.issn.1009-2137.2018.06.017. 
11. Smadja NV, Louvet C, Isnard F, et al. Cytogenetic study in multiple myeloma at diagnosis: comparison of two techniques. Br J Haematol. 1995;90(3):61924. https://doi.org/ 10.1111/j.1365-2141.1995.tb05593.x.

12. Brigaudeau C, Trimoreau F, Gachard N, et al. Cytogenetic study of 30 patients with multiple myeloma: comparison of 3 and 6 day bone marrow cultures stimulated or not with cytokines by using a miniaturized karyotypic method. Br J Hematol. 1997;96(3):594-600. https://doi.org/ 10.1046/j.13652141.1997.d01-2073.x.

13. Chng WJ, Kumar S, Vanwier S, et al. Molecular dissection of hyperdiploid multiple myeloma by gene expression profiling. Cancer Res. 2007;67(7):2982-9. https://doi.org/ 10.1158/0008-5472.CAN-06-4046.

14. Hervé MRF, Geneviève A-L. A, et al. Report from the European Myeloma Network on interphase FISH in multiple myeloma and related disorders. Haematologica. 2012;97(8):1272-7. https://doi.org/ 10.3324/haematol.

15. Committee CA-cAHO, Leukemia and Lymphoma Group SoH, Chinese Medical Association, Lijuan C, Gang A. Multiple Myeloma Genetic Testing Experts Consensus. Chinese Journal of Medical Genetics. 2019;36(2):99-102.

16. Abaza HM, Youssef SR, Saad AA, et al. Detection of $14 q 32$ rearrangements in multiple myeloma, using simultaneous FISH analysis combined with immunofluorescence. Hematol Oncol Stem Cell Ther. 2015;8(2):56-63. https://doi.org/ 10.1016/j.hemonc.

17. Hanamura I, Stewart JP, Huang Y, et al. Frequent gain of chromosome band 1q21 in plasma-cell dyscrasias detected by fluorescence in situ hybridization: incidence increases from MGUS to relapsed myeloma and is related to prognosis and disease progression following tandem stem-cell transplantation. Blood. 2006;108(5):1724-32. https://doi.org/ 10.1182/blood-2006-03-009910.

18. Zojer N, Konigsberg R, Ackermann J, et al. Deletion of 13q14 remains an independent adverse prognostic variable in multiple myeloma despite its frequent detection by interphase fluorescence in situ hybridization. Blood. 2000;95(6):1925-30.

19. zhinan Z, ti S. Hematology Diagnosis and Efficacy Standards Third Edition. Science Press.2007. p. 232-5.

20. TRIMOREAU BRIGAUDEAUC. F, GACHARD N. Cytogenetic study of 30 patients with multiple myeloma: comparison of 3 and 6 day bone marrow cultures stimulated or not with cytokines by using a miniaturized karyotypic method. Br J Haematol. 1997;96(3):594-600. https://doi.org/ 10.1046/j.13652141.1997.d01-2073.x.

\section{Figures}
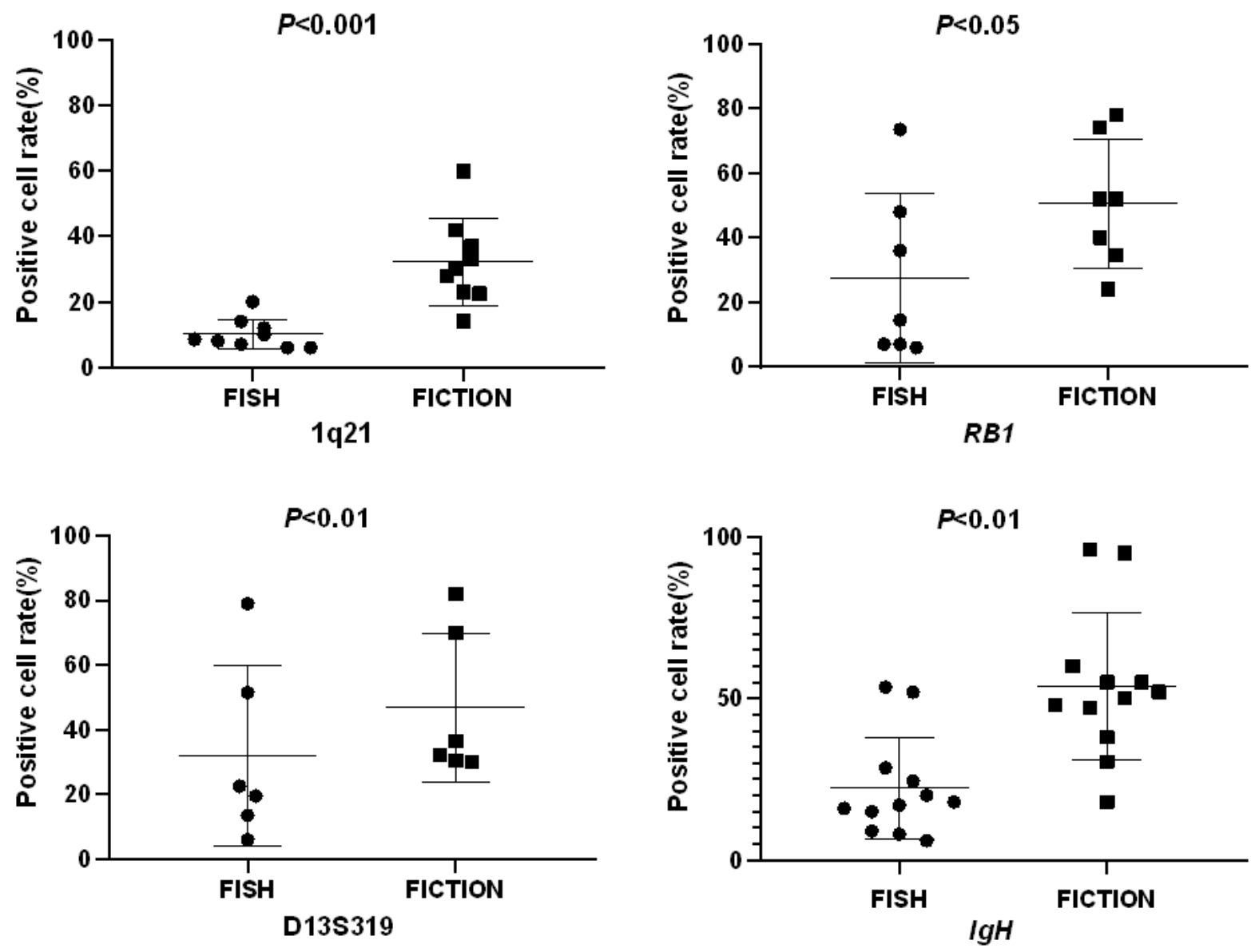

Figure 1

Page 9/11 
Positive rates of 15 samples detected both by fluorescence immunophenotyping and interphase cytogenetics as a tool for the investigation of neoplasms (FICTION) and fluorescence in situ hybridisation (FISH). P53 specimens were insufficient for analysis. The differences between 1q21, RB1, D13S319, and IgH were statistically significant $(P=0,0.038,0.004$, and 0.001 , respectively).

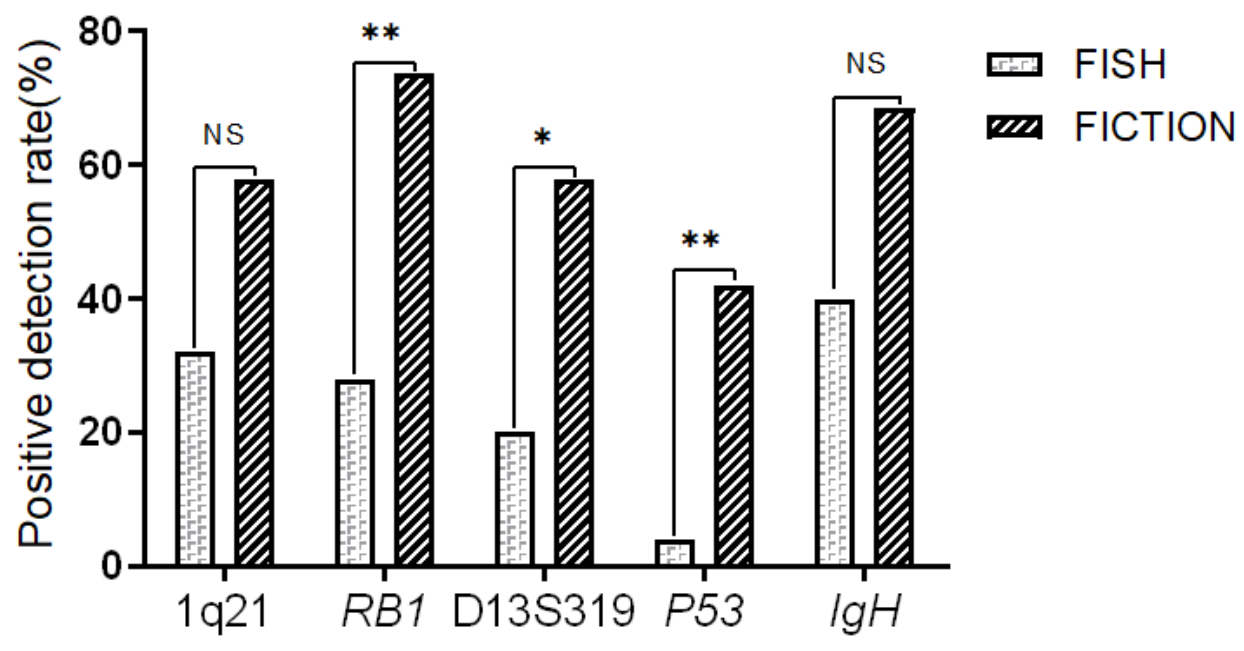

Figure 2

Abnormal detection rates of each probe. ( $\left.{ }^{*} \mathrm{P}<0.05 \rrbracket^{\star \star *} \mathrm{P}<0.01 \rrbracket^{\star \star \star} \mathrm{P}<0.001\right)$. FISH: fluorescence in situ hybridisation; FICTION: fluorescence immunophenotyping and interphase cytogenetics as a tool for the investigation of neoplasms.

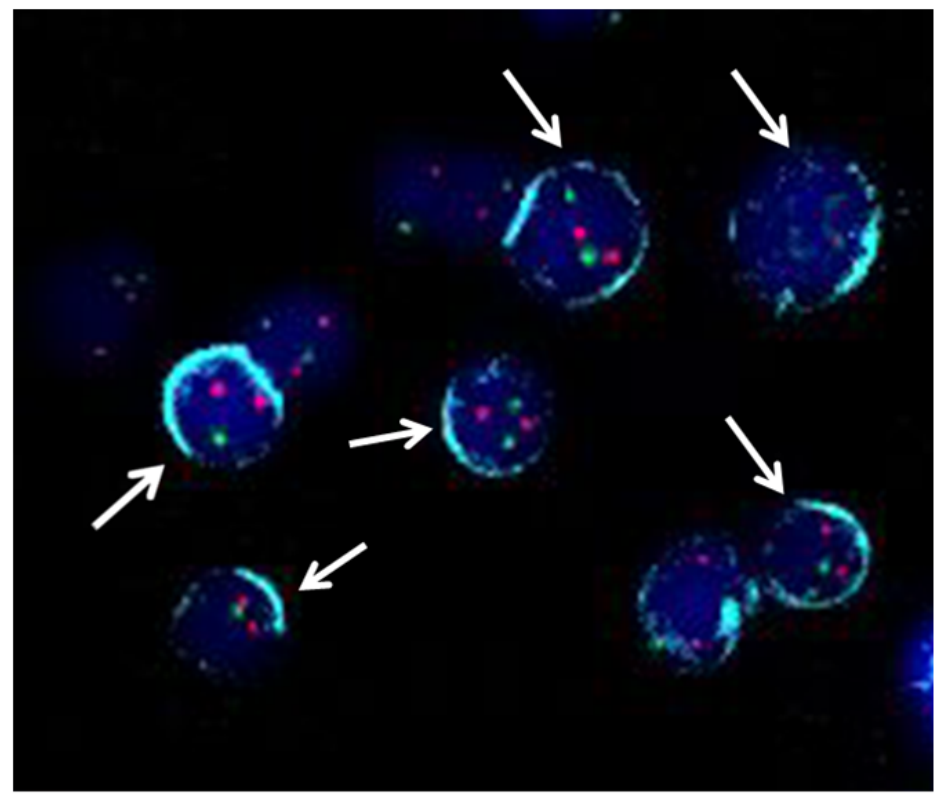

$\mathrm{a}$

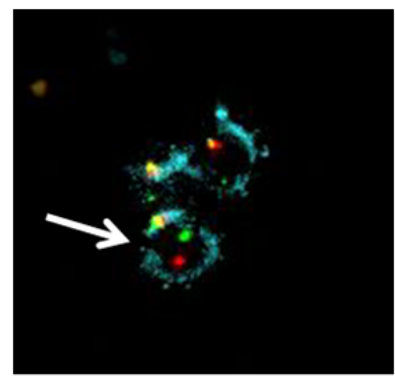

b

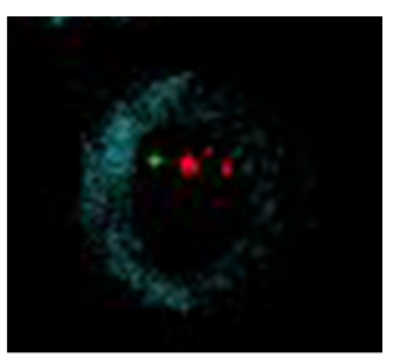

C

Figure 3 
Results of fluorescence immunophenotyping and interphase cytogenetics as a tool for the investigation of neoplasms (FICTION). a. Arrows indicate plasma cells. 2R2G denotes D13S319/ P53 normal; 2R1G denotes D13S319 normal and P53 deletion. b. Arrows indicate plasma cells with IgH break-apart (101R1G). c. Plasma cells with 1q21 amplification and RB1 deletion (3R1G).

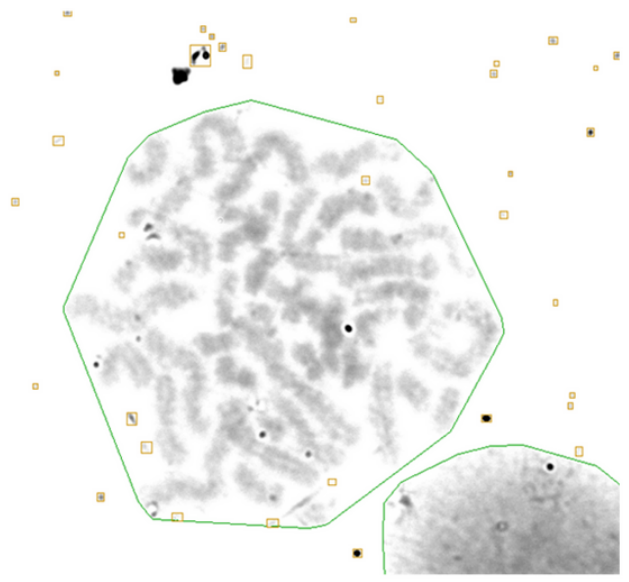

a

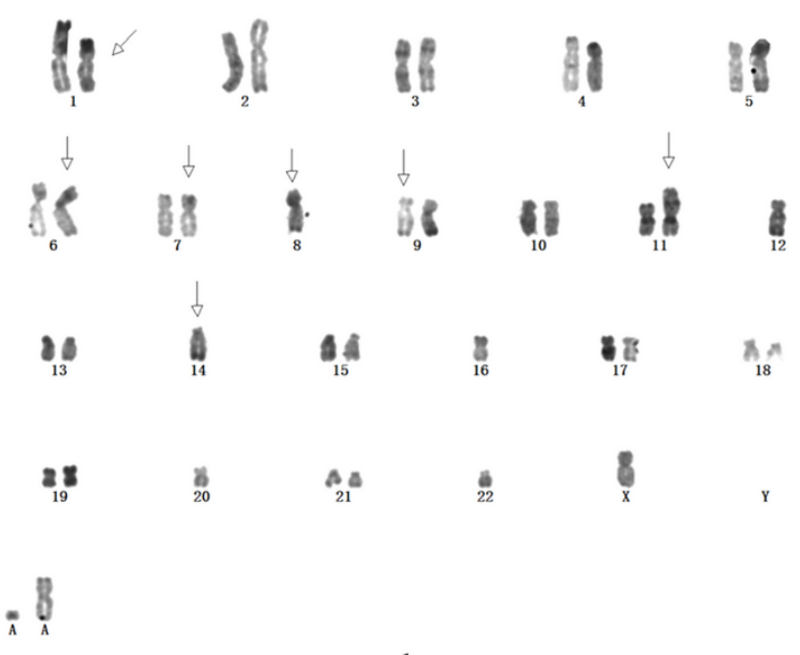

$\mathrm{b}$

Figure 4

Karyotype quality significantly increased in improved conditions in patient No. 3. a. Failure of karyotype analysis in routine culture. b. Improvement of chromosome quality in improved culture conditions, 41,X,-X, del(1)(p11p22), ins(6)(p11),? inv(7)(q22q36),-8,add(8)(p23), add(9)(p13), add(11) (p15),-12,-14, add(14)(q32),-16,-20,-22,+mar1,+mar2.

\section{Supplementary Files}

This is a list of supplementary files associated with this preprint. Click to download.

- renamedcea64.xls 